\title{
EL CONOCIMIENTO EN LA ECONOMÍA GLOBAL Y COLOMBIANA*
}

\author{
GERMÁN SÁNCHEZ PÉREZ $Z^{* *}$, LEONARDO DUARTE*** \& MARIO BLANCO***** \\ UNIVERSIDAD MILITAR NUEVA GRANADA
}

Recibido/ Received/ Recebido: 18/10/2012 - Aceptado/ Accepted / Aprovado: 03/09/2013

\begin{abstract}
Resumen
En este trabajo se muestra que el conocimiento juega un papel fundamental en el desempeño de las economías modernas y que Colombia presenta una gran brecha tecnológica en comparación con los países desarrollados y aún con los países Iberoamericanos. Se muestra que un incremento del $0.5 \%$ del progreso tecnológico aumentaría el crecimiento del PIB per cápita colombiano en un $2.0 \%$ en 5 años. Si tenemos en cuenta que las diferencias internacionales en el uso del conocimiento -en las capacidades innovadoras, en las fuentes y usos de las innovaciones, en las estrategias corporativas y condiciones institucionales- es de fundamental importancia para explicar tanto la contribución de cada país al comercio internacional, como las diferencias internacionales en los niveles de ingreso (Dosi et al, 1993), siendo la situación de la economía colombiana preocupante en este sentido.
\end{abstract}

Palabras clave: Crecimiento económico, Conocimiento, Cambio técnico, Progreso tecnológico.

\section{KNOWLEDGE IN GLOBAL AND COLOMBIAN ECONOMY}

\begin{abstract}
This article demonstrates that knowledge plays a fundamental role in modern economies performance and Colombia has a large technological gap compared to develop countries and even compared to Ibero American countries. This article shows that a $0.5 \%$ increase in technological progress would increase Colombian per capita GDP growth in $2.0 \%$ during 5 (five) years. Considering that international differences in knowledge use - innovative skills, innovation sources and uses, corporative strategies and institutional conditions are fundamental to explain both, each country contribution to international trade and international differences in income levels (Dosi et al, 1993), Colombian economy situation is critical in this sense.
\end{abstract}

Keywords: Economic growth, Knowledge, Technical change, Technological progress.

* Este documento hace parte del proyecto de investigación "conocimiento y crecimiento colombiano", financiado por la Universidad Militar Nueva Granada, 2011. Además de los autores, hacen parte del grupo de investigación el matemático Edgar Malagón, los estudiantes Jennifer Rincón, Aydée Higuera, y la joven investigadora Angélica Buitrago. Los autores agradecen la participación y valiosa colaboración de cada uno de ellos. La responsabilidad por los errores y omisiones son exclusivas de los autores.

** Candidato a doctor en Ciencias economía, Magister en Economía y Economista, docente investigador Universidad Militar Nueva Granada. Correo electrónico: german.sanchez@unimilitar.edu.co, gsanchezpe1@yahoo.com.

**** Matemático, Master en Economía. Docente-investigador Universidad Nacional de Colombia y Consultor. Correo electrónico: lduartev@unal.edu.co.

***** Profesional en Relaciones Internacionales y Estudios políticos y estudios de Maestría en Relaciones y Negocios Internacionales. Correo electrónico: marioalejandrobn@hotmail.com. 


\title{
O CONHECIMENTO NA ECONOMIA GLOBAL E COLOMBIANA
}

\author{
Resumo
}

\begin{abstract}
Neste trabalho se mostra que o conhecimento tem um papel fundamental no desempenho das economias modernas e que a Colômbia apresenta uma grande brecha tecnológica em comparação com os países desenvolvidos e ainda com os países Ibero-americanos. Mostra-se que um incremento de $0,5 \%$ do progresso tecnológico aumentaria o crescimento do PIB per capita colombiano em $2,0 \%$ em 5 anos. Se consideramos que as diferenças internacionais no uso do conhecimento - nas capacidades inovadoras, nas fontes e usos das inovações, nas estratégias corporativas e condições institucionais - é de fundamental importância para explicar tanto a contribuição de cada país no comércio internacional como as diferenças internacionais nos níveis de rendimento (Dosi et ao, 1993), sendo a situação da economia colombiana preocupante neste sentido
\end{abstract}

Palavras chave: Crescimento econômico, Conhecimento, Mudança técnica, Progresso tecnológico.

Sánchez, G., Duarte, L. \& Blanco, M. (2013) El conocimiento en la economía global y colombiana. En: Revista de la Facultad de Ciencias Económicas de la Universidad Militar Nueva Granada. rev. fac.cienc.econ, XXI (2)

JEL: H49, L70, O14, O31.

\section{Introducción}

Una característica fundamental de las economías es el cambio. En este sentido, desde finales del siglo XX diversos autores, desde diversas disciplinas, han observado que algunas economías y sociedades -USA y algunos países europeos principalmente- comenzaron a sufrir cambios importantes relacionados con los avances del conocimiento, la ciencia y la tecnología, que se ven materializados en la actividad económica a través de la innovación, la revolución informática, la globalización, las instituciones, las formas de organización; y en diversas formas en la sociedad y la cultura misma. Estos cambios económicos y sociales comenzaron a ser claramente identificados desde mediados del siglo pasado e inspiraron nuevos términos para definir tales cambios. Así, por ejemplo, Drucker (1957) acuñó el término sociedad del conocimiento, el trabajo y el trabajador del conocimiento; Touraine (1969) y Bell (1973) utilizaron el término economía y sociedad posindustrial; y Machlup (1962) empleó el término industria del conocimiento. Para finales del siglo XX, cuando los cambios fueron más evidentes, se acuñan nuevos términos para denominar los cambios del sistema capitalista, como economía del aprendizaje (Lundavall \& Johnson, 1994), nueva economía y nuevo ciclo industrial (Mandel, 1996), sociedad red o informacional (Castells, 1999), economía digital (Departamento de comercio de USA, 1998), sociedad basada en el conocimiento (OCDE, 1996), entre otros.

Es claro, sin embargo, que independientemente del término utilizado todos los trabajos resaltan y coinciden en que los nuevos cambios del capitalismo son impulsados por la revolución tecnológica basada en la información, el conocimiento, y el capital humano como fuerza productiva. En este marco, y conscientes de los cambios, algunas de las economías más avanzadas del mundo han implementado políticas que impulsan de manera clara el aprovechamiento del conocimiento como factor clave del crecimiento. USA, por ejemplo, desde el gobierno Clinton, asumió construir una economía digital estimulada por la revolución de la información; la Unión Europea se propuso convertirse en "la economía basada en el conocimiento más competitiva y dinámica del mundo" (Lisbon European Council, 2000), y otros países como Irlanda, Finlandia, Suecia y Holanda, Canadá, Australia, Nueva Zelanda y Corea del sur, han asumido el objetivo de la transformación deliberada de sus economías hacia la sociedad del conocimiento. 
Latinoamérica no ha sido ajena a los cambios y en algunos países se ha empezado a implementar desde finales del siglo XX algunas políticas, aunque parciales y desarticuladas, que buscan incentivar el uso del conocimiento en la actividad productiva. Un caso de un país Latinoamericano es el colombiano, que implementó un nuevo modelo de desarrollo desde comienzos de la década de 1990 y ha adoptado una serie de medidas que buscan incentivar el uso del conocimiento ${ }^{1}$. En este documento se abordan dos preguntas: (i) En un mundo cada vez más globalizado, ¿dónde se ubica Colombia en el contexto internacional en materia del conocimiento?; y (ii) ¿Cuál sería el impacto sobre el crecimiento económico colombiano si aumenta el progreso tecnológico?

Aparte de esta presentación e introducción, el documento se divide en dos partes. En la parte I, se evalúa y se ubica el uso del conocimiento en Colombia frente a los países más desarrollados del mundo y los países latinoamericanos. En la parte II, se realiza un ejercicio econométrico que muestra los efectos sobre el crecimiento colombiano del incremento del progreso tecnológico; $y$, finalmente se presenta las conclusiones.

\section{Metodología}

Para responder la pregunta ¿dónde se ubica Colombia en el contexto internacional en materia del conocimiento?, se hace una revisión crítica comparativa entre Colombia y un conjunto de países de los principales indicadores que miden el conocimiento. Los indicadores que se analizan son la relación de la inversión en actividades de investigación y desarrollo y crecimiento; los niveles de inversión estatal y privada en actividades de ciencia, tecnología e innovación; sectores, ejecución y financiación de estas actividades; el uso que se le da a estos recursos; el número de patentes ${ }^{2}$ colombianas en relación con los diferentes países del mundo de diferentes oficinas de patentes a nivel mundial; además de encuestas empresariales y sociales que sirvan para aclarar la situación actual del país en materia de la aplicación del conocimiento. Adicionalmente, se tienen en cuenta indicadores sobre recursos humanos, la educación en los diferentes países, así como el número de publicaciones científicas. Para medir los efectos de un incremento del progreso tecnológico sobre el crecimiento colombiano se formula y estima, teniendo en cuenta la información y las series disponibles, un modelo de co-integración y luego se proyecta el PIB per cápita para un periodo de cinco años-desde 2011 a 2015-.

Teniendo en cuenta que es alrededor de la mitad de la década de los 90 cuando se intensifica el uso de TIC's en el mundo y, por lo tanto, la aplicación del uso del conocimiento en la economía, y de acuerdo con los indicadores disponibles, se toman datos que van desde 1990 hasta el presente. Los indicadores provienen de fuentes como el Observatorio Colombiano de Ciencia y Tecnología OCyT (2010), la Red de Indicadores de Ciencia y Tecnología Iberoamericana e Interamericana RICyT (2011), la Comisión Económica Para América Latina CEPAL (2008; 2009), el Banco Mundial y sus organismos (BIRF, 2003; Banco Mundial, 2011; KAM, 2012), la Organización para la Cooperación y el Desarrollo Económico OCDE (2009), el Global Innnovation Index (2012), el Observatorio del Caribe Colombiano (2011), Colciencias (2008), el Ministerio de Educación Nacional (2010), el Departamento Nacional de Planeación DNP (2006; 2008), el Banco de la república (2011), y el Departamento Nacional de Estadística (DANE). Las series utilizadas para estimar los modelos econométricos son tomadas del Banco de la República y el DANE.

1 González \& Ángulo (2006), citan una serie de documentos oficiales donde el gobierno central coloca al conocimiento como elemento central y esencial del crecimiento económico. Muestran, de igual manera, que tales formulaciones no se corresponden con la realidad debido a las recurrentes bajas tasas de inversión en ciencia y tecnología, y una concepción política basada en el poder de mercado como elemento que guía de la manera más eficiente los efectos de la C\&T sobre el crecimiento.

2 Una patente se entiende como un contrato en el que el Estado reconoce los derechos de un invento a una persona u organización durante un tiempo definido. En dicho contrato "se le otorga al Inventor el derecho exclusivo de impedir que otros fabriquen, utilicen o vendan el invento patentado durante un periodo de tiempo fijo, a cambio de que éste presente al público los detalles del invento" (Universidad Javeriana, 2010). 


\section{Colombia en el contexto global del co- nocimiento}

\subsection{Aspectos conceptuales sobre la economía del conocimiento}

Aunque el conocimiento ha estado presente en los diferentes sistemas de producción de la humanidad, es en el capitalismo donde se resalta su importancia; en especial a partir de la segunda mitad del siglo XX con trabajos como los de Schumpeter (1942), Solow (1956, 1957), Romer (1990), entre otros. Para Morín, por su parte, "El conocimiento es sin duda un fenómeno multidimensional en el sentido que, de manera inseparable, a la vez es físico, biológico, cerebral, mental, psicológico, cultural, social" (Morín, 2002, 20). Según sus posibilidades de expresión, el conocimiento puede dividirse en conocimiento explícito y conocimiento tácito (Polanyi, 1958, 1978; citado en Vilaseca \& Torrent, 2005). El conocimiento explícito es aquel que se puede expresar en forma escrita, de forma sistemática, es decir aquel que se puede codificar y por tanto puede hacerse perdurar en el tiempo, así como ser transferido fácilmente a otros individuos o mantenerse como un secreto comercial para mantener la ventaja de la posesión de ese conocimiento con respecto a otras empresas por ejemplo ${ }^{3}$. El conocimiento tácito, en cambio, es difícil de reproducir, es adquirido por la experiencia y el tiempo, se considera un activo invisible y depende de las formas en que el trabajador o el empresario resuelven problemas y cumplen sus objetivos (Fernández, Montes, Pérez, \& Vásquez, 1998).

La relación conocimiento y crecimiento económico, es reconocida en la literatura económica desde los clásicos a finales del siglo XVIII. Smith (1776), por ejemplo, resaltó el papel que juega el conocimiento en el bienestar de una nación; y desde la heterodoxia Schumpeter (1911), por ejemplo, resaltó la importancia de la innovación en el crecimiento económico. En la actualidad varios autores e instituciones resaltan la relación positiva entre el conocimiento, el crecimiento y el desarrollo. El Banco Interamericano de desarrollo, por ejemplo, señala que:

"La capacidad de una sociedad para producir, seleccionar, adaptar, comercializar y usar el conocimiento es crucial para lograr un crecimiento económico sostenido y mejorar los estándares de vida de la población. El conocimiento se ha convertido en el factor preponderante de desarrollo económico" (BIRF, 2003).

\section{Según la $\mathrm{OCDE}^{4}$ :}

"Incorporar el conocimiento en las funciones económicas estándar de un país no es una tarea fácil, debido a que este factor desafía algunos principios económicos como el de la escasez. El conocimiento y la información tienden a ser abundantes, lo que escasea es la capacidad de usarlos en una forma significativa" (OECD, 1996, 11).

En Colombia, para entidades como Colciencias:

"La generación de conocimiento y sus múltiples aplicaciones son elementos centrales para el desarrollo económico y social de las sociedades contemporáneas y son básicos para responder a los requerimientos y necesidades de la sociedad" (Colciencias 2008, 6).

En este mismo sentido, Planeación Nacional (DNP, 2006) señala, siguiendo a Romer, que el conocimiento es un bien público y que, por lo tanto, se caracteriza por no tener rivalidad, es decir que si una persona o empresa consume conocimiento, esto no disminuye las posibilidades de consumo de otras personas, el costo marginal de agregar un nuevo usuario es cero; $y$, en segundo lugar, por la ausencia de exclusión, lo que quiere decir que una vez que se produce el conocimiento nadie puede ser excluido de sus beneficios ${ }^{5}$.

\footnotetext{
3 Un ejemplo es la receta y proceso de fabricación de la Coca-Cola, receta que a pesar de la gran difusión de la gaseosa, se ha mantenido secreta por 120 años logrando así ser uno de los secretos comerciales mejor guardados.

Organización Para la Cooperación y El Desarrollo Económico, también conocida como OECD por sus siglas en inglés.

Excepto en los casos de los secretos comerciales.
} 
En síntesis, la teoría y la empírea muestran que existe una relación directa entre la generación, difusión y aplicación del conocimiento y el desempeño económico, y la economía del conocimiento se encarga de estudiar dicha relación. El conocimiento se manifiesta de varias formas en la actividad productiva $y$ puede impulsarse a través del esfuerzo por la investigación y el desarrollo tecnológico; el número de centros de investigación y su calificación; los graduados de carreras científicas, ingenierías, técnicas y tecnológicas; la relación de la empresa con la academia y el Estado; la educación de calidad, en el sentido que la formación de capital humano. En este sentido, todas las actividades que impulsan el avance, distribución y aplicación del conocimiento en la actividad productiva son elementos centrales de las estrategias de desarrollo tecnológico de los países y de las empresas (CEPAL, 2008, 33).

\subsection{El conocimiento en Colombia y en el mun- do: un análisis comparativo}

Colombia es un país de desarrollo medio, con un crecimiento promedio anual en el siglo XX alrededor del $4.7 \%$. Y aunque éste crecimiento no ha sido el resultado de una política de Estado que busque incentivar el desarrollo, uso, difusión y aprovechamiento interno del conocimiento, éste último ha jugado un papel importante en el crecimiento económico a través del progreso tecnológico, sobre todo entre la década de 1920 y finales de la década de 1970, periodo a partir del cual la economía colombiana empieza a mostrar una tendencia decreciente del crecimiento hasta finales de siglo (Sánchez, 2011). Con el cambio de modelo adoptado desde comienzos de la década de 1990 -se pasó de un modelo proteccionista, de sustitución de importaciones, a uno de economía abierta, de apertura de la economía-, Colombia ha direccionado, aunque tímidamente, su política económica hacia el conocimiento desde finales del siglo pasado.

En un mundo cada más vez integrado e impulsado por la materialización del conocimiento en la actividad productiva, es importante mirar, en un contexto global, cómo se encuentra el país en materia del conocimiento en términos comparativos. De este aspecto nos ocupamos en seguida. Por la mis- ma amplitud del concepto y sus múltiples formas de impactar la economía, medir los efectos del conocimiento sobre una economía no es fácil y no existe un único indicador que mida y recoja los efectos de dicha relación. Enseguida se mira los indicadores más reconocidos que nos aproxime a un entendimiento de los efectos del conocimiento sobre el crecimiento económico en el mundo, en general, y en Colombia, en particular, haciendo énfasis en la situación colombiana en el contexto global.

\subsection{Recursos financieros}

La Investigación y Desarrollo (I+D) puede entenderse como un trabajo creativo que de forma sistemática busca incrementar el volumen de los conocimientos humanos, culturales y sociales y el uso de estos conocimientos para derivar nuevas aplicaciones en todos los campos de la ciencia y tecnología, involucrando investigación básica, aplicada y el desarrollo experimental (OECD, 2002); incluye, además, la enseñanza y la formación de científicos e ingenieros, y los servicios científicos y tecnológicos. Por esta razón, la inversión en I\&D y su relación con el crecimiento del PIB se ha convertido en uno de los indicadores más usados para mirar los efectos del conocimiento sobre el crecimiento.

La Ilustración 1 muestra que efectivamente existe una relación positiva entre el gasto en $\mathrm{I}+\mathrm{D}$ y el crecimiento económico en el mundo. En efecto, los países que más invierten en $\mathrm{I}+\mathrm{D}$ son los que muestran los mayores PIB per cápita. Países de alto crecimiento como, por ejemplo, Suecia invierte más del $4 \%$ del total de su PIB en I+D, Israel ronda el 5\%, USA se acerca al 3\%, y Japón y Finlandia superan el 3\%. En contraste, los países latinoamericanos no llegan a superar el uno por ciento de inversión en en $\mathrm{I}+\mathrm{D}$ en relación a su PIB per cápita, exceptuando a Brasil, quedando entre los países que menos invierten en $\mathrm{I}+\mathrm{D}$.

Colombia se encuentra entre los países que menos invierten en $\mathrm{I}+\mathrm{D}$ en el mundo y posee, dentro de la muestra de países de la llustración 1, uno de los PIB per cápita más bajos. En efecto, los niveles del gasto en actividades de Ciencia, Tecnología e innovación (ACTI) e I+D han sido históricamente bajos en el 
Ilustración 1. PIB per cápita y gasto en Investigación y Desarrollo (2000-2004) ${ }^{6}$

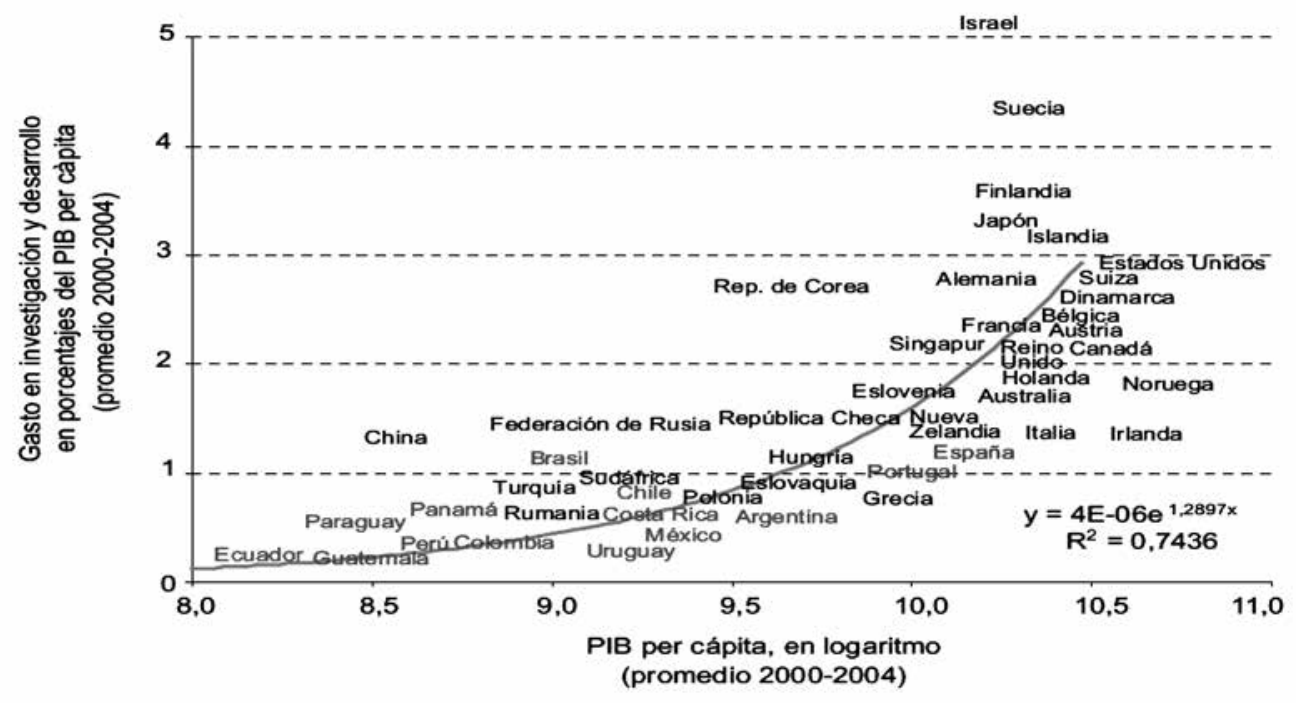

país, y a pesar de la adopción del modelo de desarrollo de apertura desde la década de 1990, la inversión en ACTI no ha alcanzado el $0.6 \%$ (ilustración 2) del PIB, niveles de inversión actuales que son muy bajos en términos comparativos internacionales.

Conclusiones similares se obtienen cuando se observa el gasto en Ciencia y Tecnología en términos absolutos, por país y grupo de países. Las disparidades en el crecimiento económico en el mundo, al igual que las disparidades en inversión en $\mathrm{I}+\mathrm{D}$, son altas. En efecto, Estados Unidos, Alemania Francia y el Reino Unido representan el $66 \%$ del gasto total mundial en $\mathrm{I}+\mathrm{D}$, mientras el gasto en $\mathrm{I}+\mathrm{D}$ que hace Iberoamérica es de tan sólo el 3,3\% del gasto total mundial en el primer periodo que va de 1990-1995, y aunque su participación crece hasta el $4 \%$ en el segundo periodo -2000 a 2003-, continúa siendo muy bajo. Llama la atención países como China -que posee una de las mayores tasas de crecimiento en las últimas décadas-, donde la inversión en $\mathrm{I}+\mathrm{D}$ pasó de un 2.4\% entre 1990-1995 hasta alcanzar un 8.4\% entre 2000-2003, un ejemplo notable de aumento en la inversión en I+D y crecimiento económico.
La conclusión general es que Colombia es uno de los países del mundo desarrollado y de América Latina, que menos gasta en ACTI y menos invierte en $\mathrm{I}+\mathrm{D}$, lo que podría explicar, en parte, por qué la productividad del trabajo en el país se encuentra tan lejos de la productividad del trabajo de éstos países. En efecto, los 10 países que más invirtieron en $\mathrm{I}+\mathrm{D}$ entre 1990-2010 están entre los más desarrollados y presentan una productividad alta y creciente, Colombia se encuentra en clara desventaja frente a los países de la muestra y está por debajo del promedio mundial.

\subsection{Recursos humanos}

La base de un buen sistema nacional integrado de Ciencia y Tecnología (CyT), son los recursos humanos y una adecuada política Nacional de Ciencia y Tecnología (CEPAL, 2008). Así mismo, existe una relación positiva entre el número de personas dedicadas a la investigación y el crecimiento de las economías: los países con mayor crecimiento tienen más investigadores per cápita y son más dinámicas en temas relacionados con la ciencia la tecnología y

6 Fuente: Elaboración propia con datos RICyT (2011). 
Ilustración 2. Gasto en ACTI e I+D en Colombia como porcentaje del PIB, 1995-20107

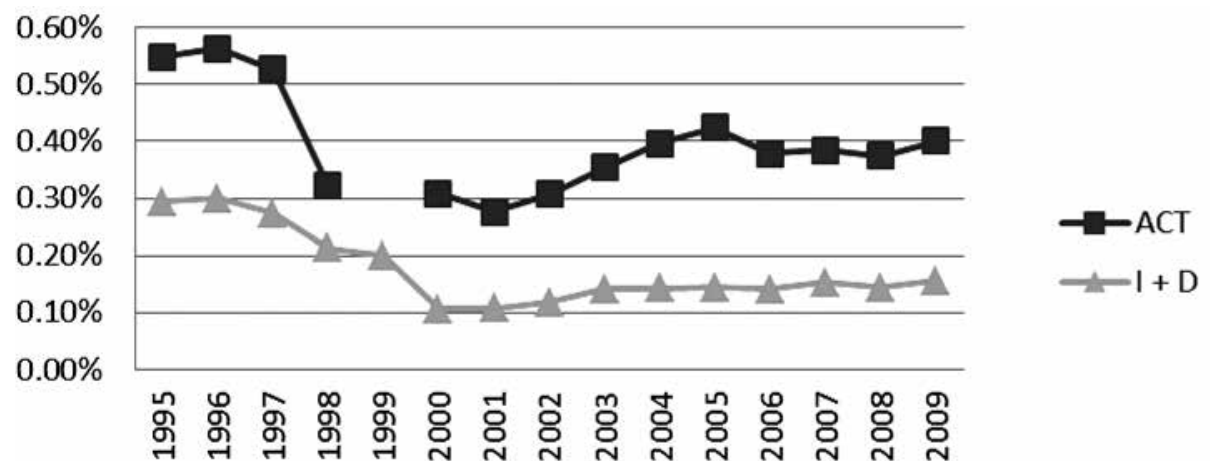

la innovación. Como se aprecia dentro del círculo verde, los países de América Latina -incluyendo a Colombia- se encuentran en el cuadrante inferior izquierdo, siendo los más atrasados tanto en número de investigadores como en el PIB per cápita.

Del personal total dedicado en el mundo a la I+D (Ilustración 3), se observa una gran concentración, siendo Estados Unidos, China y Japón los tres países que concentran más de la mitad. La Unión Europea y países como Corea del Sur cuentan con un número importante de investigadores, mientras que los países iberoamericanos se encuentran en clara desventaja con el resto del mundo. Los países en Iberoamérica que cuentan con más investigadores son España, con 161.933 para 2004, y Brasil con 157.595. Países con un alto crecimiento económico como Alemania cuentan con 470.729 investigadores y Estados Unidos con 1.335.148. Para este mismo año (2004), Colombia contaba con un total de 9.918 personas dedicada a la I+D.

En consonancia con lo que hemos venido destacando, en la llustración 4 se observa que existe una relación positiva entre el número de investigadores por millón de habitantes y el crecimiento económico. En efecto, los países de mayor crecimiento poseen igualmente un mayor número de investigadores por millón de habitantes. Es de destacar que mientras que el promedio mundial es de 1821 investigadores por millón de habitantes, en América Latina y el Caribe sólo se alcanza 484,7 investigadores; es decir, menos de la tercera parte de los investigadores del promedio mundial. Obsérvese que Colombia es uno de los países en el mundo con uno de los niveles más bajos de investigadores por millón de habitantes.

En Colombia el número de investigadores totales ha aumentado en los últimos años (Ilustración 5). En efecto, mientras que el número de investigadores totales en 1996 era de 3840, para el año 2009 esta cifra llegó a 15886, cifra que continua siendo muy baja comparado con los estándares internacionales.

Además, los investigadores en Colombia están concentrados en ciudades como Bogotá y Medellín, lo que genera una gran divergencia entre las posibilidades de investigación entre las diferentes regiones (OCyT, 2010). Bogotá, desde el año 2000, se ha mantenido con aproximadamente el 39\% de los investigadores del país, y junto con Antioquia sumaron el $55 \%$ del total para 2009.

\subsection{Educación}

Puesto que el capital humano es la base de un sistema nacional de ciencia, tecnología e innovación, disponer de una base importante y de alta calidad de Re-

\footnotetext{
7 Fuente: Elaboración propia con datos de RICyT (2011).
} 
Ilustración 3. Distribución mundial del personal dedicado a la I+D según país de origen 2004 o último año disponible ${ }^{8}$

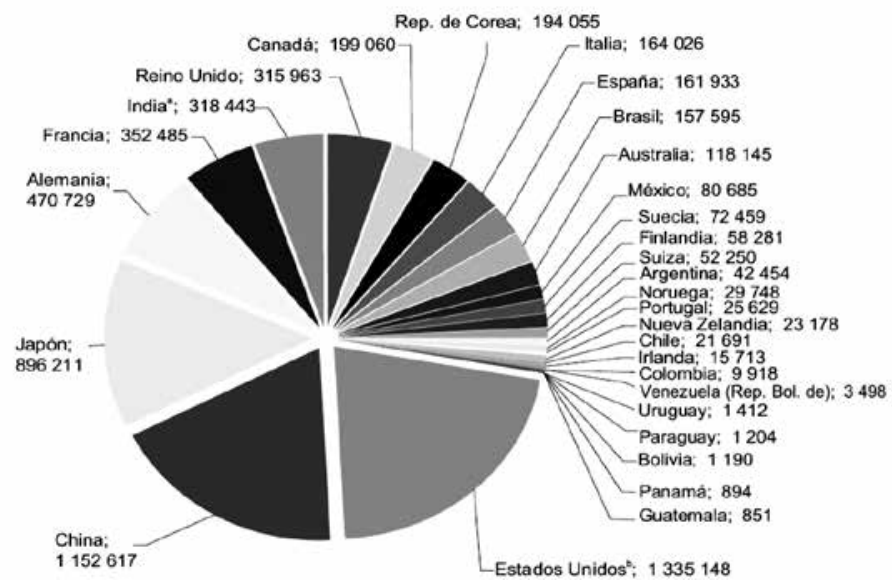

Ilustración 4. Número de Investigadores por millón de habitantes año 2008 o último disponible ${ }^{9}$

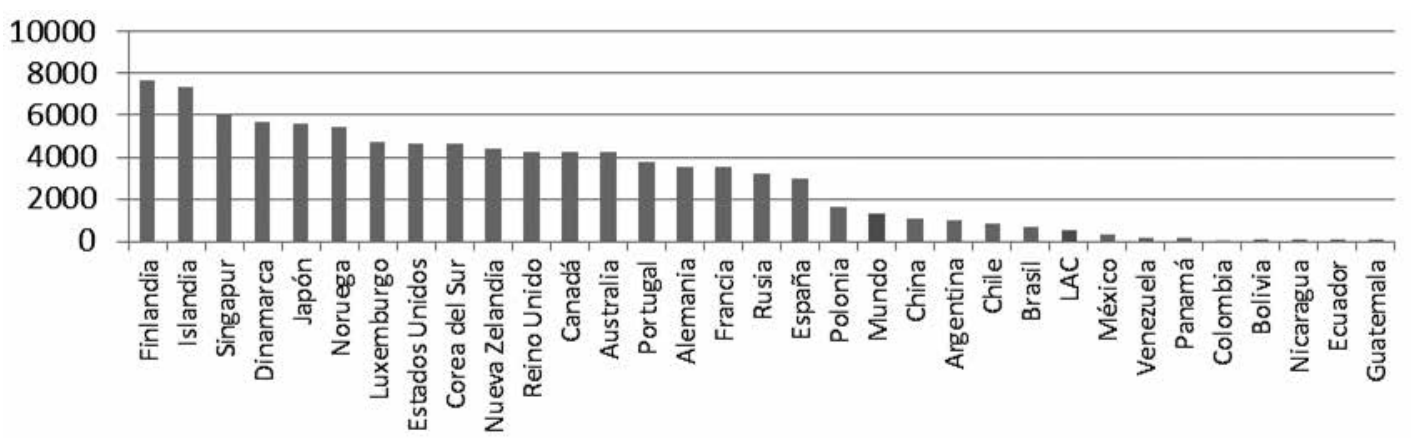

Ilustración 5. Número de investigadores Colombia (Total) ${ }^{10}$

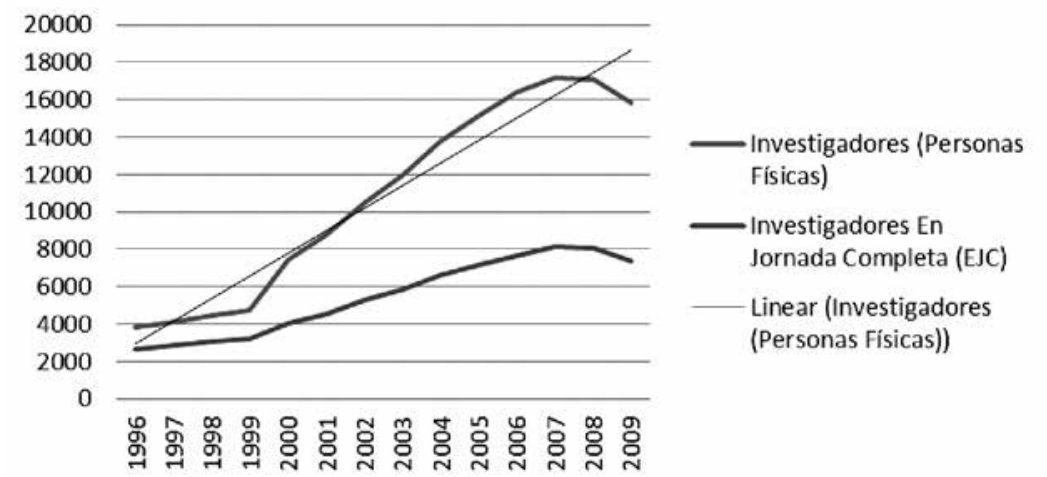

Fuente: CEPAL $(2008,35)$.

Fuente: CEPAL (2008).

Fuente: Elaboración propia con datos de RICyT (2011). 
cursos Humanos para la investigación y el desarrollo es necesario para promover la innovación. A pesar de los esfuerzos realizados en los últimos años para fomentar la educación básica y media en América Latina, las economías de mayor crecimiento muestran grandes diferencias en este aspecto. En efecto, en la prueba de Ciencias del Programa Internacional de Evaluación de Estudiantes (PISA) de la OCDE, Alemania, Japón, Shanghái, Finlandia, Corea del sur, y USA ocupan puestos relevantes entrando en los niveles más altos de la prueba; mientras que los países latinoamericanos se ubican a la derecha de la Ilustración ocupando una posición desventajosa. De los países Ibéricos, España y Portugal se encuentran en posiciones ventajosas, aunque no tan altas como otros países de la OCDE.

En cuanto a la cobertura en educación superior o terciaria (Ilustración 6), América Latina posee un bajo nivel, comparada con los países desarrollados. Colombia está por debajo del promedio iberoamericano con alrededor del $27 \%$ de cobertura en educación superior según datos de la CEPAL. España y Portugal se encuentran en posiciones más ventajosas, y es de resaltar el caso de Argentina que se ubica por encima de países como Reino Unido y Canadá con un porcentaje aproximado del $63 \%$ convirtiéndose en un ejemplo para la región.

El patrón de la formación universitaria según la disciplina científica, es un indicador importante a la hora de determinar en qué se ocupa el recurso humano de un país. En este indicador también se observa una clara diferencia entre países desarrollados como USA y Latinoamérica. En efecto, mientras en los Estados Unidos existe preferencia por las ciencias exactas, en América Latina, en general y Colombia, en particular, existe preponderancia por las ciencias sociales (CEPAL, 2008).

A pesar de la clara desventaja que posee Colombia en materia de educación en un contexto internacional, en los últimos años ha venido mejorando, pero continúa estando aún lejos de los estándares inter- nacionales. Las mejoras se observan principalmente en la cobertura de la educación básica primaria, básica secundaria, y media (Ilustración 7), debido al fomento de la educación a los estudiantes con bajos recursos en todo el país (Ministerio de Educación Nacional, 2011).

\subsection{Patentes}

En cuanto al número de patentes, los países desarrollados presentan mejores indicadores que los países de América Latina. En cuanto la eficacia en la producción de patentes por la inversión en $\mathrm{I}+\mathrm{D}$, por ejemplo, los niveles alcanzados por países desarrollados como Japón, USA, Corea del Sur, son muy superiores a los niveles alcanzados por los países de Iberoamérica (Ilustración 8).

Si miramos el coeficiente de invención, medido como el número de patentes solicitadas por cada cien mil de habitantes (Ilustración 9), también se observa los mejeros resultados presentados por los países desarrollados frente a los países Iberoamericanos. En efecto, mientras el coeficiente de invención en Estados Unidos fue cerca de 80 para el año 2009, para los países de América Latina este coeficiente, en este mismo año, fue mucho menor: en Colombia, por ejemplo, fue apenas de 0.28, en Bolivia de 0.5, Argentina de 1.60, Cuba de 0.53, y México de 0.76. La situación en España es mucho más favorable alcanzando 8.2 y en Canadá es de15.03.

\subsection{Publicaciones}

Las publicaciones en revistas científicas son un indicador de la cantidad y calidad del trabajo realizado por los investigadores y centros de investigación de los distintos países. Uno de las publicaciones más reconocidas en el mundo de los índices de publicaciones indexadas es el Science Citation Index ${ }^{11}$ (SCI), que cubre más de 3700 de las principales revistas científicas de más de 100 disciplinas diferentes (Thompson Reuters, 2012). Siguiendo este indicador, en la siguiente Ilustración se puede apreciar el

11 No confundir con el Science Citation Index Expanded también de Thompson Reuters, que Incluye alrededor de 6650 revistas con alrededor de 150 disciplinas. 
Ilustración 6. Tasa bruta de matrícula educación superior año 2004 o último disponible ${ }^{12}$

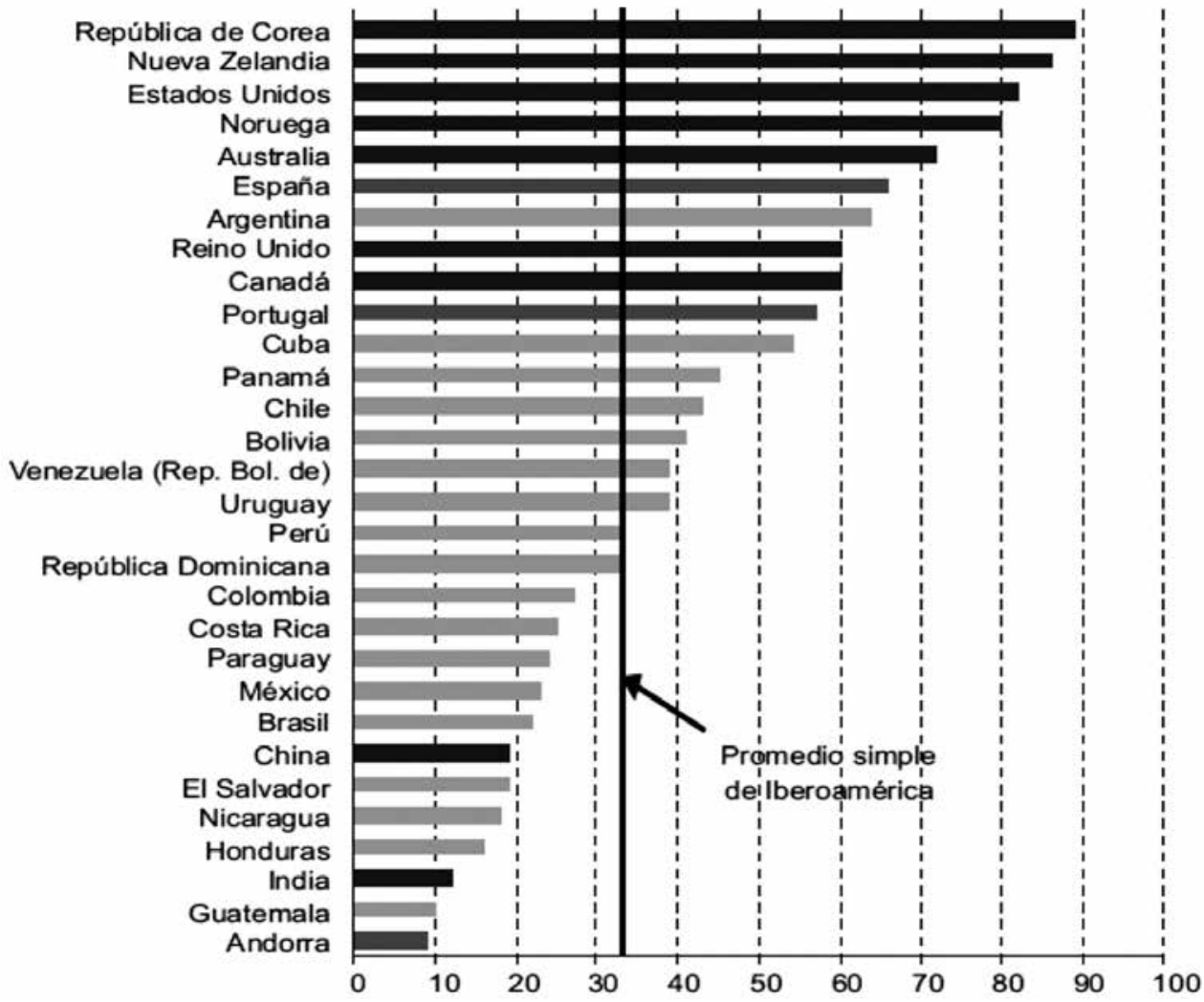

Ilustración 7. Matrícula total educación básica y media en Colombia ${ }^{13}$.

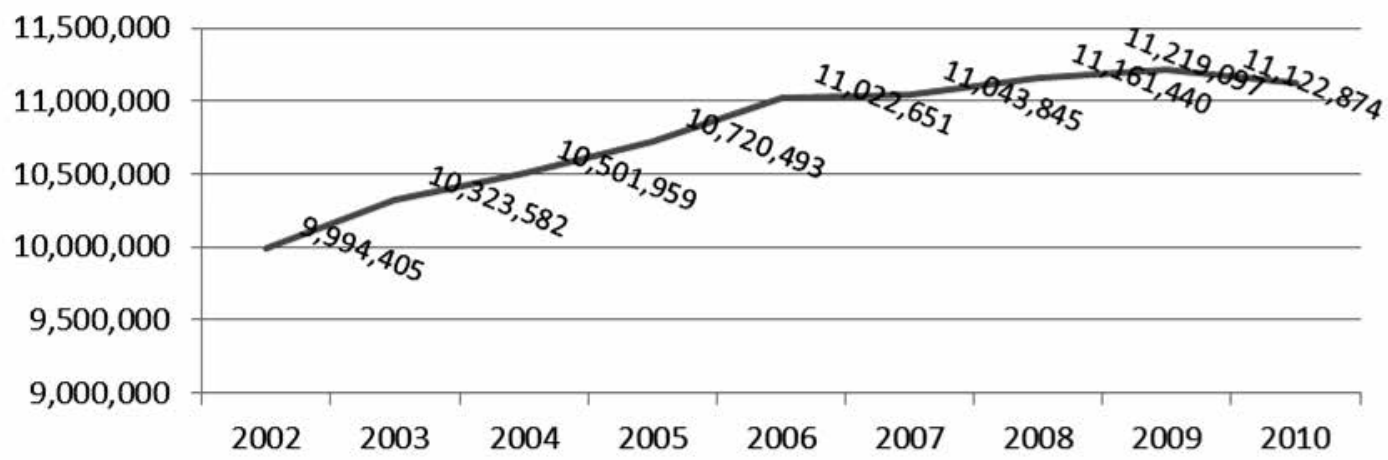

Fuente: CEPAL (2008).

Fuente: Elaboración propia con datos de Ministerio de Educación Nacional (2010). 
Ilustración 8. Eficacia de producción de patentes respecto a la inversión en I+D ${ }^{14}$

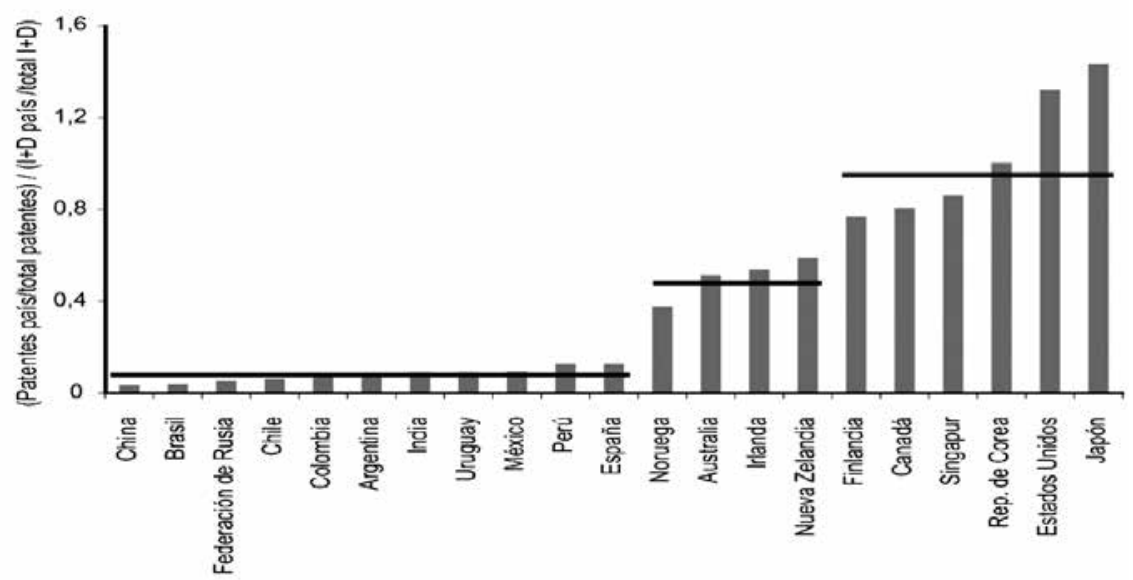

Ilustración 9. Coeficiente de invención países seleccionados año $2009^{15}$

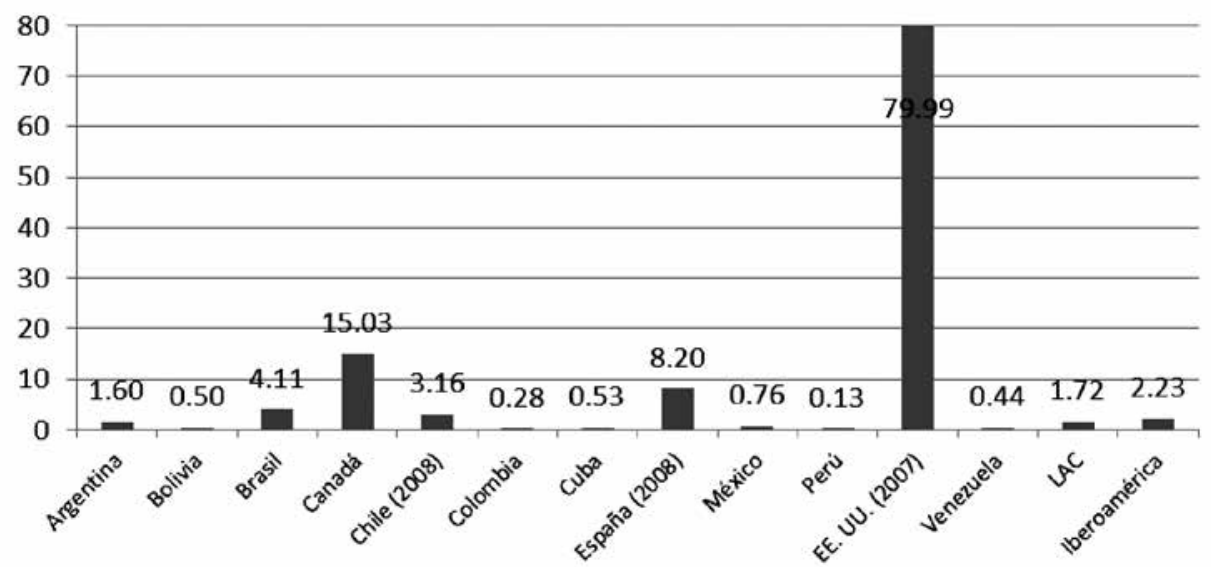

número total de publicaciones por país en el año 2009. Resalta la gran ventaja que le lleva Estados Unidos a los países de Iberoamérica.

Colombia ha avanzado en las últimas décadas en el número de publicaciones. En efecto, mientras que para 2002 el número de publicaciones colombianas era de 815 y 463 para SCI y Pascal respectivamente, para el año 2009 esta cifra llegaba a 2386 y 961, lo que representa un avance importante. Adicionalmente, Colombia ha hecho esfuerzos por implementar su propio sistema de indexación denominado $\mathrm{Pu}$ - blindex que tiene sus orígenes en 1996, cuando en el país se hizo un ejercicio de escalafonamiento de las revistas científicas. Desde este momento y por su progresiva integración al SNCyT se ha buscado que Publindex sea un sistema de indexación y resumen, un índice bibliografico y una base bibliollustración (OCyT, 2010).

La situación desfavorable de Colombia en comparación con otras economías en el mundo en materia del conocimiento, se aprecia igualmente con los índices del Banco Mundial de la Economía del Cono-

14 Fuente: CEPAL (2008).

15 Fuente: Elaboración propia con datos de RICyT (2011). 
cimiento $\mathrm{KEI}^{16}$ y $\mathrm{KI}^{17}$, los cuales representan el grado de preparación en general que tiene un país o región para competir en un contexto internacional. Como lo ilustra estos indicadores (tabla 1), Colombia se encuentra en una posición media-baja en comparación con otros países; y los países que se encuentran en su misma escala son en gran parte latinoamericanos. En efecto, de 146 países evaluados, Colombia ocupa el puesto 76 en el KEI y 67 en el KI; y en ninguno de los cuatro pilares que señala el Banco Mundial en el KEI, el país supera el puesto 64. En Iberoamérica, países como España, Portugal, México, Panamá, Brasil y Perú superan ampliamente a Colombia en el KEI.

Tabla 1. Clasificación de Colombia en los índices KEI, KI y de los cuatro pilares de la economía del conocimiento ${ }^{18}$

\begin{tabular}{|c|c|c|c|c|c|c|}
\hline & KEI & KI & EIR & $\begin{array}{c}\text { Innova- } \\
\text { ción }\end{array}$ & $\begin{array}{c}\text { Educa- } \\
\text { ción }\end{array}$ & $\begin{array}{c}\text { Inf1. } \\
\text { TIC }\end{array}$ \\
\hline Puntuación & 4,94 & 5,18 & 4,25 & 4,68 & 5,28 & 5,57 \\
\hline Posición & 76 & 67 & 80 & 73 & 70 & 64 \\
\hline
\end{tabular}

Situación similar a la observada en los índices del Banco Mundial, se presenta al analizar el Global Innovation Index, que mide la innovación globalmente mediante el estudio de indicadores relacionados con el desempeño y las condiciones que permiten la innovación en un país y clasifica a los países del mundo según sus características. En efecto, de 125 países evaluados Colombia ocupa el puesto 71 .

En resumen, en Colombia no ha habido una política de Estado dirigida al desarrollo, uso y difusión del conocimiento, ni existe una tradición en inversión en ACTeI. La tradición en el país ha sido importar tecnología en vez de desarrollarla y los montos de inversión en ACTI son muy pequeños si se comparan con los países desarrollados y se encuentra por debajo del promedio de Latinoamérica. Otro as- pecto colombiano contrario a lo observado en los países de mayor desarrollo, es la poca inversión de fuentes privadas; según el Observatorio Colombiano de Ciencia y Tecnología (2010), en el año 2010 la inversión privada para ACTI sólo alcanzó el 39.26\% del total mientras la inversión estatal fue de $51.06 \%$. Por otro lado, los trámites para invertir en ACTI dificultan crear empresa y patentar en el país. Un aspecto a resaltar es el avance interno reciente del país en su recurso humano; sin embargo, en un contexto internacional Colombia está realmente atrasada en este aspecto. La poca vocación que existe en el país hacia las actividades de investigación, sumado al poco apoyo del gobierno nacional y el sector privado a estas actividades, se refleja en el porcentaje de personas dedicadas a la investigación. En efecto, los investigadores de tiempo completo en Colombia fueron para el año 2008 sólo 0.36 por cada 1000 integrantes de la PEA, cifra que para América Latina y el Caribe alcanzó 1.03, es decir, más del doble de investigadores; y las cifras se vuelven aún más preocupantes cuando se compara con un país como Portugal donde la cifra llegó a 7.18. En cuanto a la vocacion por la innovacion, en 2004 se graduaron 80,404 profesionales en nuestro país, de los cuales el $22 \%$ cursó programas de administración, el $11 \%$ de educación y el $9 \%$ de derecho; pero en cuanto a las carreras relacionadas con la innovación las cifras son desalentadoras, ingeniería electrónica, ingeniería de telecomunicaciones y otras captaron el $3.03 \%$, ingeniería mecánica el $1.43 \%$ e ingeniería eléctrica el $0.76 \%$, matemáticas tuvo apenas el $0.2 \%$ y física el $0.1 \%$ (DNP, 2006). Otro de los problemas que se identifican para el uso del conocimiento en Colombia, es la debilidad y falta de independencia del $\mathrm{SNCT}+\mathrm{I}$, lo que se suma a la falta de formulación de políticas de Estado. Otra deficiencia que se identifica en términos comparativos internacionales, es la poca motivación para el desarrollo de actividades de CTeI, en especial por parte de los empresarios. Para 2005,

17 Fuente: Elaboración propia a partir de KAM (2012).

17 El Knowledge Economy Index (KEI), se basa en un promedio simple de cuatro subíndices que representan cuatro pilares de la economía del conocimiento: Incentivos Económicos y Régimen Institucional (Economic Incentive and Institutional Regime, EIR), Innovación y Adopción tecnológica (Innovation and Technological Adoption), Educación y Entrenamiento (Education and Training), y por último infraestructura en Tecnologías de la Información y Comunicaciones TIC (Information and Communications Technologies ICT Infrastructure) (KAM, 2012).

18 El Knowledge index es un promedio simple entre tres de los indicadores del conocimiento que conforman los 4 pilares del KEI; no se toma el EIR, se basa en Innovación y adaptación tecnológica, Educación e Infraestructura en TIC's. 
Ilustración 10. Publicaciones en SCI países seleccionados $2009^{19}$.

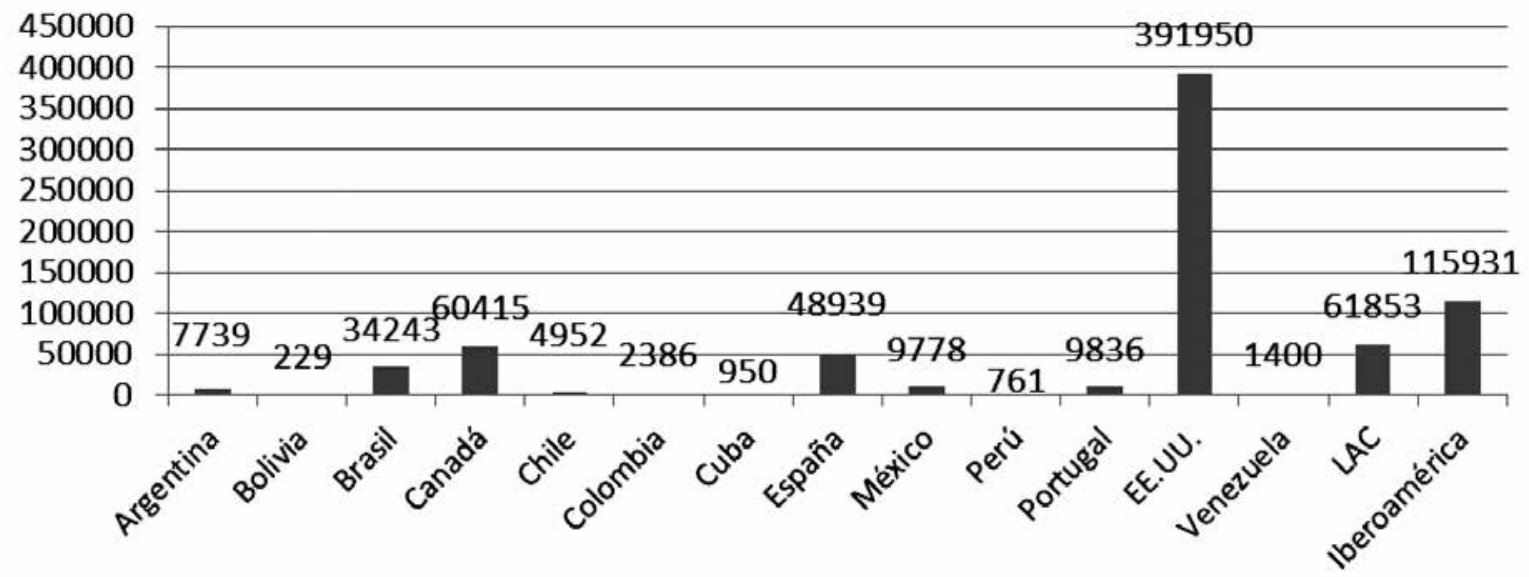

Ilustración 11. Índice Global de Innovación, Global Innovation Index ${ }^{20}$

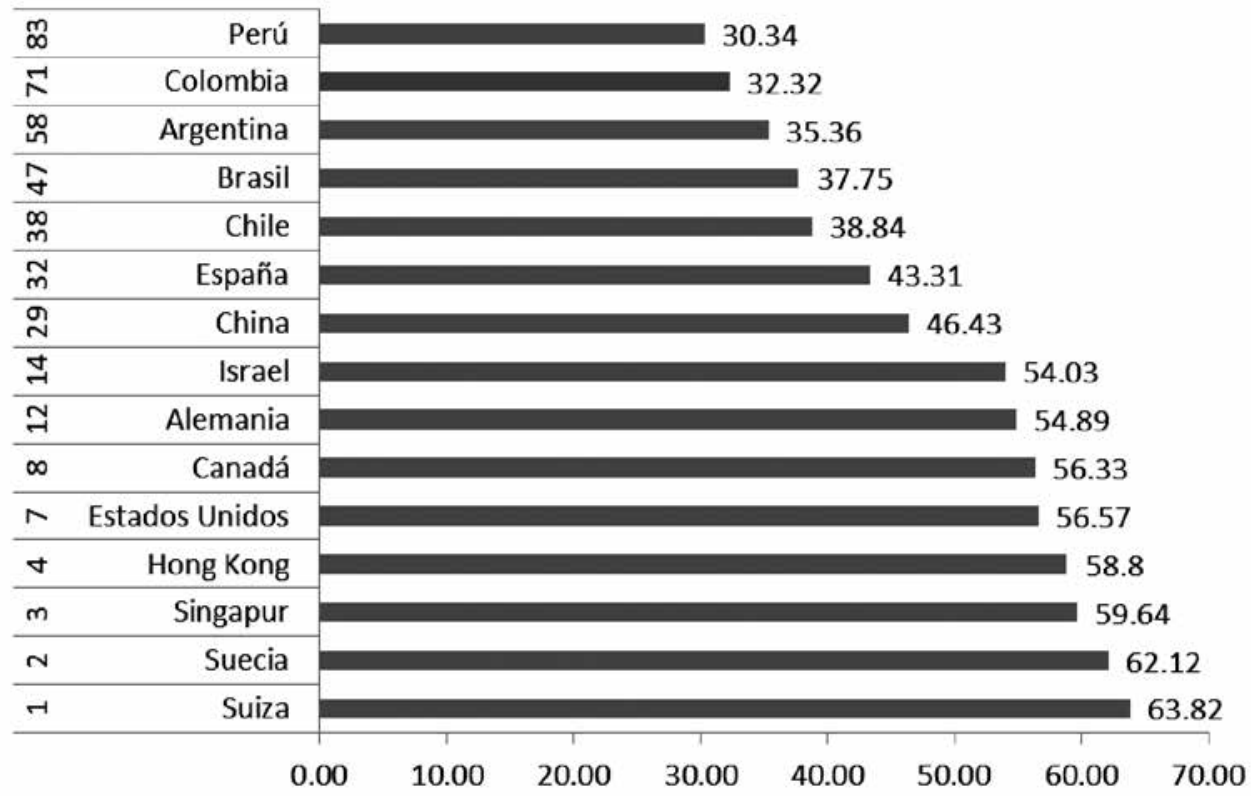

19 Fuente: Elaboración propia con datos de RICyT (2011).

20 En la parte izquierda de la Gráfica se encuentra la posición en que cada país se encuentra según el índice, y a mano derecha la puntuación obtenida.

Fuente: Elaboración propia con datos de RICyT (2011). 
Sólo el 8.3\% de las 6172 empresas encuestadas entraban en la categoría de innovadoras radicales ${ }^{21}$.

\section{El conocimiento y el crecimiento en Colombia: efectos de un cambio tecnoló- gico sobre el crecimiento}

Dos aspectos fundamentales que se muestran en la parte I de este trabajo son: primero, Colombia presenta una gran brecha tecnológica con los países desarrollados y con gran parte de los países de Iberoamérica; y, segundo, la teoría y la evidencia empírica revelan que la aplicación del conocimiento en la actividad productiva crea grandes ventajas para el crecimiento y el progreso de un país. En este contexto, surgen inquietudes como qué se ha hecho en el país en materia del conocimiento y en cuánto afectaría un incremento del cambio tecnológico el crecimiento económico colombiano. Un ejercicio de esta naturaleza justifica y da evidencia cuantitativa a la idea de que la aplicación del conocimiento en la actividad productiva crea grandes ventajas para el crecimiento y el progreso colombiano.

Para responder a estos interrogantes enseguida se presenta, primero un breve resumen del conocimiento en Colombia y, posteriormente, se formula y estima un modelo de cointegración VEC (Vector de corrección errores).

\subsection{Antecedentes del conocimiento en Colombia}

En Colombia, desde la época de la colonia se han realizado esfuerzos, aunque parciales e insuficientes, por impulsar la ciencia. En este periodo se fundaron las primeras instituciones educativas apoyados por sacerdotes jesuitas, lo que significó un impulso para el desarrollo intelectual en el nuevo continente. Más adelante hubo investigaciones derivadas de expediciones, como la expedición en busca de El Dorado, en el siglo XVI, y la expedición Botánica, en 1783, que significó que en la Nueva Granada se gestara el interés por las ciencias naturales y las ciencias exactas. Aunque no fue sino hasta 1820, con la aparición de la república, que se comienza a organizar realmente la educación en el país, con la fundación de la Universidad de Boyacá y la Universidad Central. Asimismo, se hacen algunos esfuerzos para transferir conocimiento al país con misiones de sabios que se componían de expertos e ingenieros de distintas áreas provenientes principalmente de Alemania, Francia y Bélgica, además de la inclusión de modelos educativos de corte inglés que promovían la educación laica (DNP, 2006).

Posteriormente, a mediados del siglo XIX, la república inició una Misión Corográfica con el fin de identificar la geografía del país, así como sus recursos naturales, cuencas hidrográficas y demás para así poder medir las posibilidades que tenía cada una de las entidades locales del país. Por esa misma época, una misión alemana estuvo en el país con el fin de mejorar los métodos pedagógicos, lo que conllevó a la fundación de la Universidad Nacional, en 1867, y otras escuelas normales (DNP, 2006). A comienzos del siglo XX, se fundan las primeras universidades departamentales, y se promueven carreras de ingeniería, agronomía, minas, medicina, y se formaron institutos autónomos para resolver problemas locales.

No obstante, antes de 1968 no se puede hablar de una organización institucional de ciencia y tecnología en Colombia, sino de una serie de procesos que venían gestando la posibilidad de que esto se diera en el país (DNP, 2006). En efecto, fue 1967, en la cumbre de presidentes en Punta del Este (Uruguay), que se acuerda llevar a cabo reformas en Latinoamérica para fomentar el comercio, modernizar las economías, aumentar la eficiencia y la productividad, y la creación de políticas de Ciencia y Tecnología. En

21 Entre las razones que explican este fenomeno está la falta de confianza que los empresarios colombianos le tienen a participar en redes de innovación. Según la Encuesta Nacional de Percepción de la Ciencia y la Tecnología (DNP, 2006), el 77\% de las empresas cree que de acuerdo a su experiencia invertir en ciencia y tecnología es un buen negocio; sin embargo, sólo el $41 \%$ de las empresas encuestadas cree que participar en redes de innovación es importante, debido a que no asocian la participación en procesos de innovación, ciencia y tecnología como algo que pueda traer desarrollo para ellas. 
Colombia, esto llevó a la creación de Colciencias en 1968 como un fondo para la ciencia, encargado de coordinar, difundir y ejecutar proyectos de investigación científica. En este mismo periodo, las universidades colombianas hacen esfuerzos por crear algunas de las primeras maestrías y en el mundo entraron en auge nuevas tecnologías como la biotecnología, la informática, nuevos materiales, la microelectrónica, y la alta química en los años 80. Las universidades colombianas comienzan a incentivar la investigación y en 1986 se crean los primeros cuatro programas doctorales en la Universidad Nacional. Para 1987 se propone la creación de un ministerio para la CyT, y en 1988 se revisa el estado del arte de la investigación en el país. Como resultado de esta investigación, en 1990 se crearon las directrices para la inversión en $\mathrm{CyT}^{22}$ y se crea el Sistema Nacional de Ciencia y Tecnología (SNCyT) en $1991^{23}$, como "un sistema abierto, no excluyente, del cual forman parte todos los programas, estrategias y actividades de ciencia y tecnología, independientemente de la institución pública o privada o de la persona que los desarrolle" (Decreto 585 de 1991). Así mismo, Colciencias deja de hacer parte del Ministerio de Educación Nacional y se convierte en un organismo del Departamento Nacional de Planeación.

En 1993 se conforma una misión de sabios, con el fin de evaluar el sistema de educación, que resaltó el grave atraso del país en esta materia en un contexto internacional. Como resultado de esta evaluación, se gesta el Plan para la endogenización de la ciencia y la tecnología, que tenía como objetivo formar nuevos centros de investigación, nuevos investigadores, y la apropiación de la CyT por parte de la sociedad, en especial niños y jóvenes. En 1994 se elabora el primer documento Conpes y en 1995 se crea el Sis- tema Nacional de Innovación (SNI) que busca generar cambios en la estructura empresarial. Durante este periodo, el SNCyT, con la introducción del modelo SNI, cambia de nombre y se convierte en el Sistema Nacional de Ciencia Tecnología e Innovación $\left(\mathrm{SNCTe}{ }^{24}\right)$ y recibió importantes recursos gracias a créditos del BID y del SENA ${ }^{25}$, que estaba obligado a usar parte de sus recursos en proyectos de CyT. En 1996 surge Publindex como el Índice de Publicaciones Científicas Colombianas, más adelante se crea la base de datos GrupLAC ${ }^{26}$ y $\mathrm{CvLAC}^{27}$ para los grupos de Investigación, y para las hojas de vida y experiencia de los investigadores respectivamente. En 1998 se crea Maloka como el primer centro interactivo de CyT de Suramérica, y en 1999 se crea el Observatorio Colombiano de Ciencia y Tecnología con el fin de medir el avance en esta materia en el país, como parte del SNCyT. En 2002, Colciencias crea las Comisiones Departamentales de Ciencia y Tecnología (CODECyT), que buscaban incentivar la investigación y el desarrollo de la CyT en los departamentos. Para 2006, 29 departamentos habían logrado que estas comisiones operaran regularmente (DNP, 2006). En el año 2004, el Consejo Nacional de Ciencia y Tecnología $\left(\mathrm{CNCy}^{28}\right)$ crea el programa de Centros de Investigación de Excelencia, con lo que se busca incursionar en distintas áreas ${ }^{29}$ novedosas en el país.

\subsection{Modelo de co-integración}

De acuerdo con la series disponibles en Colombia y teniendo en cuenta que los efectos del conocimiento sobre el crecimiento se manifiestan en el mediano y largo plazo, los efectos sobre el crecimiento colombiano pueden captarse a través de un modelo de corrección de errores -VEC, por sus siglas en inglés-.

\footnotetext{
Mediante la Ley 29 de 1990.

Mediante el Decreto Ley 585 de febrero de 1991.

También conocido por las siglas SNCT +I

La Ley 344 señala que el SENA deberá destinará el 20\% de los recursos para programas de competitividad y desarrollo tecnológico productivo.

26 Grupo de Investigación Latinoamérica y el Caribe.

27 Curriculum Vitae Latinoamérica y el Caribe.

28 Cabeza del Sistema Nacional de Ciencia, Tecnología e Innovación.

29 Como biodiversidad y recursos genéticos; biotecnología e innovación agroalimentaria y agroindustria; enfermedades infecciosas prevalentes en áreas tropicales, materiales avanzados y nanotecnología, modelamiento y simulación de fenómenos y procesos complejos, y cultura, instituciones y desarrollo.
} 
En efecto, a partir de una función de producción del tipo Cobb-Douglas podemos formular y estimar -siguiendo a Sánchez (2011); Greco (2002), y Obstfeld \& Rogoff (1996) un modelo como el siguiente. Con $\mathrm{Y}=\mathrm{PIB} ; \mathrm{K}=$ capital; $\mathrm{L}=\mathrm{PEA} ; \mathrm{A}=$ factor o parámetro tecnológico que aumenta la eficiencia laboral, $\alpha=$ participación de $\mathrm{K}$ en $\mathrm{Y}$, (con $0<\alpha<1), \mathrm{t}+1=$ un periodo cualquiera, rendimientos marginales decrecientes del capital y rendimientos constantes de escala- tenemos

$$
Y_{t+1}=K_{t+1}^{\alpha}\left[A_{t+1}^{(1 / 1-\infty)} L_{t+1}\right]^{1-\infty}
$$

Modelo VEC:

$$
\begin{aligned}
& \Delta y_{t}=\theta_{11} \Delta y_{t-1}+\theta_{12} \Delta k_{t-1}+\pi_{1}\left(y_{t-1}-\alpha k_{t-1}-\gamma-\beta_{t}\right)+\varepsilon_{1 t} \\
& \Delta k_{t}=\theta_{21} \Delta y_{t-1}+\theta_{22} \Delta k_{t-1}+\pi_{2}\left(y_{t-1}-\alpha k_{t-1}-\gamma-\beta_{t}\right)+\varepsilon_{2 t}
\end{aligned}
$$

Los coeficientes $\theta 11, \theta 12, \theta 21, \theta 22$ reflejan el impacto de corto plazo de cambios en las variaciones en $\mathrm{t}-1 ; \pi 1, \pi 2$ representan las velocidades de ajuste a un desequilibrio entre el producto por trabajador $y$ el capital por trabajador y sus determinantes de largo plazo; $\alpha, \gamma$ y $\beta$ son los coeficientes de largo plazo. Con $\pi 1, \pi 2 \leq 0$. Donde,

$$
\beta=1-\alpha \delta
$$

Despejando $\delta$, obtenemos:

$$
\delta=\beta 1-\alpha
$$

Y como $\delta=\ln 1+$ ga, solucionando la ecuación y despejando obtenemos la tasa de progreso tecnológico (ga) (ver Sánchez, 2011):

$$
\mathrm{ga}=e \delta-1
$$

En este marco de análisis, el crecimiento de la productividad del trabajo está relacionado con el crecimiento del capital por trabajador y la tasa de crecimiento del factor de productividad; y una vez corrido el VEC, podemos estimar la tasa de crecimiento por periodo de A o progreso tecnológico ga (ecuación 4). Conocida la tasa del progreso tecnológico podemos proyectar el PIB per cápita tanto con este valor estimado como con una variación (incremento) del mismo.
Las series del producto por trabajador y capital por trabajador no son estacionarias, son integradas de orden 1 - I(1) - y están co-integradas. Empleando el método de Johansen (1988, 1995), y una vez realizadas las pruebas formales correspondientes, se encontró que el VEC que se debe estimar para todo el periodo de análisis, 1970-2010, debe contener constante, tendencia, dos variables dummy y un rezago. Los datos se toman del Banco de la República y el DANE, y los modelos se estimaron en CATS.

Una vez realizadas las estimaciones se encontró que el crecimiento promedio anual del cambio tecnológico es del $0.8 \%$ y el crecimiento anual promedio de la productividad total de los factores (PTF) es del $0.6 \%$, para el periodo 1970-2010.

En la tabla 2 se presenta el PIB per cápita proyectado, desde el 2011 hasta el 2015, con la tasa de progreso tecnológico $(0.8 \%)$ resultante del VEC estimado, y el PIB per cápita que se obtendría si el cambio tecnológico se incrementará en medio punto porcentual (de $0.8 \%$ a $1.3 \%$ ); es decir, el crecimiento del PIB per cápita proyectado sin y con variaciones del progreso tecnológico, respectivamente.

Los resultados muestran que un incremento del cambio tecnológico afecta de manera positiva el crecimiento económico colombiano. En efecto, si el cambio tecnológico se incrementa en $0.5 \%$, el PIB per cápita se incrementa en promedio anual un $0.41 \%$, lo que daría como un resultado un incremento acumulado en el crecimiento del PIB per cápita al cabo de 5 años del $2.03 \%$.

\section{Conclusiones}

Algunas de las conclusiones generales que se pueden extraer del presente documento son las siguientes:

- A pesar que el progreso tecnológico ha jugado un papel importante en la explicación del crecimiento colombiano (Sánchez, 2011; Robinson y Urrutia, 2007), en el país no se ha adoptado una política económica de Estado clara encaminada al desarrollo, uso e implementación del conocimiento en la actividad productiva. En consecuencia, la economía colombiana no pre- 
Tabla 2. Proyección del PIB cápita sin y con un incremento del progreso tecnológico, 2011-2015³0

\begin{tabular}{|c|c|c|c|}
\hline Año & $\begin{array}{c}\text { Proyección PIB per } \\
\text { cápita inicial }\end{array}$ & $\begin{array}{c}\text { Proyección PIB per cápita } \\
\text { incrementado el cambio } \\
\text { tecnológico en } \mathbf{0 . 5} \%\end{array}$ & Diferencia \\
\hline 2011 & $3,0 \%$ & $3,3 \%$ & $0,31 \%$ \\
\hline 2012 & $2,9 \%$ & $3,4 \%$ & $0,43 \%$ \\
\hline 2012 & $2,8 \%$ & $3,3 \%$ & $0,45 \%$ \\
\hline 2014 & $2,7 \%$ & $3,1 \%$ & $0,43 \%$ \\
\hline 2015 & $2,6 \%$ & $3,0 \%$ & $0,41 \%$ \\
\hline & \multicolumn{2}{|c|}{ Acumulado } & $2.03 \%$ \\
\hline
\end{tabular}

senta las características propias de una economía basada en el conocimiento.

- Todos los indicadores que miden el uso del conocimiento en las economías, muestran que Colombia se encuentra muy por debajo de los países con mayores niveles de desarrollo y por debajo de la mayoría de los países de Iberoamérica. En efecto, en términos comparativos Colombia presenta bajos niveles de Inversión en Ciencia, Tecnología e Innovación (CTeI); a pesar de los avances recientes, existe un rezago en la capacidad científica y tecnológica; insuficiente recurso humano para actividades de $\mathrm{CTeI}$ y falta de vocación científica; falta de institucionalización del SNCT + I y promoción de sus políticas en lugares alejados y sectores estratégicos para el país para el largo plazo; poca motivación para el desarrollo de actividades de $\mathrm{CTeI}$, en especial por parte de los empresarios; $y$, falta de conexión entre las actividades generadoras de conocimiento y la actividad productiva -relación academia-empresa y entre empresas-.

- Es clara la brecha tecnológica que en términos comparativos internacionales presenta el país. Situación preocupante si tenemos en cuenta que en un mundo globalizado el conocimiento es el factor clave del crecimiento, la competitividad, y el ingreso per cápita.
- Nuestros resultados muestran que un incremento de medio punto porcentual del progreso tecnológico que presenta la economía colombiana entre 1970-2010, incrementaría en $2.03 \%$ el PIB per cápita colombiano entre 2011-2015.

- La teoría y la evidencia muestran que aplicación del conocimiento en la actividad productiva crea grandes ventajas para el crecimiento y el progreso de un país; sin embargo, es necesario crear un marco institucional que incentive el desarrollo, uso y materialización del conocimiento en la actividad productiva. El conocimiento es la fuerza impulsora de las sociedades modernas $y$, por lo tanto, una sociedad que no mira hacia el conocimiento es una sociedad que no mira hacia el futuro.

\section{Referencias}

Banco de la República (2011). Colombia, Informe de Balanza de Pagos. Bogotá.

Banco Mundial (2011). Base de datos Banco Mundial. Recuperado el 25 de Noviembre de 2011, de http://data.worldbank.org/

Bell, D. (1973). El advenimiento de la sociedad postindustrial. Madrid: Alianza Editorial.

BIRF (2003). Construir Sociedades de Conocimiento: Nuevos Desafíos para la Educación Terciaria. Washington: Quebecor World Bogotá S.A.

Castells, M. (1999). La era de la información: economía, social y cultura. México: Siglo XXI editores.

CEPAL (2008). Espacios Iberoamericanos: La Economía Del Conocimiento. Santiago.

30 Cálculos de los autores. 
CEPAL (2009). Innovar Para Crecer, Desafíos y oportunidades para el desarrollo sostenible e inclusivo en iberoamérica. Santiago de Chile: Naciones Unidas.

Colciencias (2008). Colombia Construye y Siembra Futuro, Política Nacional de Fomento a la Investigación y la Innovación. Bogotá.

Decreto 585 de 1991. Recuperado el 05 de Noviembre de 2011, de Colciencias: http://www.colciencias.gov.co/normatividad/ decreto-585-de-1991

Departamento de Comercio de EE.UU (1998). The emerging digital economy.

DNP (2006). Visión Colombia II Centenario Fundamentar el crecimiento y el desarrollo social en la ciencia, la tecnología y la innovación. Bogotá: Imprenta Nacional de Colombia.

DNP (2008). Departamento Nacional de Planeación. Recuperado el 10 de Julio de 2011, de DNP Portal WEB - Indicadores de Ciencia, Tecnología e Innovación: http://www.dnp.gov.co/PortalWeb/ Programas/Comercioindustriadesarrolloempresarial/CienciayT ecnolog\%C3\%ADaeInnovaci\%C3\%B3n/Estad\%C3\%ADsticas/ tabid/237/Default.aspx

Drucker, P. (1957). The landmarks of Tomorrow. Harper and Row.

Fernández S., Montes, J., Pérez B. \& Vásquez, C. (1998) Acumulación, Naturaleza e Imitación del Conocimiento Tecnológico: Una Revisión de La Literatura. En: Investigaciones Europeas de Dirección y Economía de la Empresa, Vol. 4, No. 1: 11-34.

Global Innovation Index (2012). Obtenido de http://www.globalinnovationindex.org/. Fecha consulta: 10 de marzo.

González, J. \& Ángulo, M. (2006). "La ciencia y la tecnología en el desarrollo económico colombiano. La brecha entre el discurso y la realidad".

Greco (2002). El crecimiento económico colombiano en el siglo XX. Banco de la república. Fondo de Cultura Económica.

Johansen, S. (1988). Statistical analysis of cointegration vectors. En: Journal of economic dynamics and control, 12: 231-254.

Johansen, S. (1995). Likelihood-based inference in cointegrated vector autoregressive models. Oxford University Press.

KAM (2012). Knowledge for Development. Obtenido de http://www. worldbank.com/kam. Fecha consulta: 5 de mayo del 2013.

Lisbon European Council (2000). Presidency Conclusions. Obtenido de: http://www.lisboncouncil.net

Lundavall, B. \& Jhonson, B. (1994). The learning economy. En: Journal of Industry Studies, 4(2), 23-42.

Machlup, F. (1962). The production and distribution of knowledge in the United States. Princeton University Press, 416p.

Mandel, M. (1996). The triumph of the emergence of a new economy. Business Weel.

Ministerio de Educación Nacional (2010). Centro Virtual de Noticias Sobre Educación. Recuperado el 25 de Julio de 2011, de UN impulsa el primer Parque Científico y Tecnológico del país: http:// www.mineducacion.gov.co/cvn/1665/w3-article-249745.html

Ministerio de Educación Nacional (2011). Mineducación anuncia educación gratuita para 2012. Recuperado el 04 de Noviembre de 2011, de Al día con las noticias: http://www.mineducacion. gov.co/observatorio/1722/article-280181.html

Ministerio de Educación Nacional (2010). Ministerio de Educación Nacional. Recuperado el 15 de Noviembre de 2011, de Estadísticas del sector educativo: http://menweb.mineducacion. gov.co/seguimiento/estadisticas/principal.php?consulta = mat total\&nivel $=2$

Morín, E. (2002). El método III. El conocimiento del conocimiento. Traducción de Ana Sánchez, cuarta ed. Madrid: Cátedra.

Observatorio Colombiano de Ciencia y Tecnología (2010). Observatorio Colombiano de Ciencia y Tecnología. Recuperado el 10 de Junio de 2011, de Indicadores de Ciencia y Tecnología Colombia: http://ocyt.org.co/html/archivosProyectos/COLOMBIA_2010.pdf

Obstfeld, M. \& Rogoff, K. (1996). Foundations of international macroeconomics. MIT Press, Cambridge (Ma).

OCC. Observatorio del Caribe Colombiano (2011). Recuperado el 05 de Noviembre de 2011, de Sistema de Indicadores de la región Caribe Colombiana: http://www.ocaribe.org/sid/sid.php

OCyT. Observatorio Colombiano de Ciencia y Tecnología (2010). Recuperado el 10 de Junio de 2011, de Indicadores de Ciencia y Tecnología Colombia: http://ocyt.org.co/html/archivosProyectos/ COLOMBIA 2010.pdf

OECD (1996). The Knowledge-Based Economy. Recuperado el 05 de Julio de 2011, de http://www.oecd.org/dataoecd/51/8/1913021. pdf

OECD (2002). Manual de Frascati. Obtenido de www.micinn.es/ stfls/MICINN/.../ManuaFrascati-2002 sp.pdf

OECD (2009). The PISA 2009 profiles by country/economy. Retrieved Noviembre 07, 2011, from http://stats.oecd.org/ PISA2009Profiles/

RICyT (2011). Red de Indicadores de Ciencia y Tecnología - Iberoamericana e Interamericana. Recuperado el Julio - Diciembre de 2011, de Indicadores por País, Comparativos y Reportes: http://www.ricyt.org/

Robinson, J. \& Urrutia, M. (2007). Economía colombiana del siglo XX. Un análisis cuantitativo. Fondo de Cultura Económica, Banco de la República, Colombia.

Romer, P. (1990). Endogenous Technical Change. En: Journal of Political Economy, Vol. 98, núm. 5: 71-102.

Sánchez, G. (2011). Conocimiento y crecimiento colombiano: una aproximación a través del progreso tecnológico y el cambio técnico. En: Revista Facultad de Ciencias Económicas: Investigación y Reflexión, XIX (2).

Schumpeter, J. (1911). The Theory of Economic Development. Cambridge, EE. UU.: Harvard University Press.

Schumpeter, J. (1942). Capitalism, socialism and democracy. New York, Mc Graw Hill.

Smith, A. (1776). Una investigación sobre la naturaleza y causas de la riqueza de las naciones. Londres.

Solow, R. (1956). A contribution to the theory of economic growth. En: Quarterly journal of economics 70.

Solow, R. (1957). Technical change and the aggregate production function. En: Mueller, Holt, Rinehart y Winston (eds), Reading in macroeconomics, N.Y, 1967.

Thompson Reuters, (2012). Thompson Reuters. Recuperado el 08 de Enero de 2012, de http://thomsonreuters.com/products services/ science/science products/a-z/science citation index/

Touraine, A. (1969). La sociedad post-industrial. Barcelona: Ariel. Universidad Javeriana (08 de 03 de 2010). ¿Qué es una patente?

Vilaseca, J. \& Torrent, J. (2005). Principios de la Economía del Conocimiento. Madrid: Pirámide. 